\title{
Diversidad y microestructura de quitones (Mollusca: Polyplacophora) del Caribe de Costa Rica
}

\author{
Cedar I. García-Ríos ${ }^{1}$ \& Migdalia Álvarez-Ruiz ${ }^{2}$ \\ 1. Departamento de Biología, Universidad de Puerto Rico en Humacao, Humacao, Puerto Rico 00792; \\ cedar.uprh@gmail.com \\ 2. Departamento de Biología, Universidad de Puerto Rico en Ponce, Ponce, Puerto Rico 00732; \\ migdalia.alvarez1@upr.edu
}

\author{
Recibido 20-I-2010. Corregido 21-IX-2010. Aceptado 19-X-2010.
}

\begin{abstract}
Diversity and microstructure of quitons (Mollusca: Polyplacophora) from the Caribbean of Costa Rica. The polyplacophorans of the coral reef on the Caribbean coast of Costa Rica have been insufficiently studied. The examination of coral rubble accumulated in the shallow sublitoral waters on four collection stations in Provincia Limón revealed a higher diversity of chitons than was documented. From the country eight species were previously known: Ischnochiton erythronotus (C.B. Adams 1845); Ischnoplax pectinata (Sowerby 1840); Stenoplax boogii (Haddon 1886); S. purpurascens (C.B. Adams 1845); Acanthopleura granulata (Gmelin 1791); Chiton marmoratus Gmelin 1791; C. tuberculatus Linnaeus 1758 and Acanthochitona rhodea (Pilsbry 1893). This study added five more species that are reported here for the first time: Callistochiton portobelensis Ferreira 1976; Ischnochiton kaasi Ferreira 1987; I. pseudovirgatus Kaas 1972; Acanthochitona balesae Abbott 1954 and Cryptoconchus floridanus (Dall 1889). Rev. Biol. Trop. 59 (1): 129-136. Epub 2011 March 01.
\end{abstract}

Key words: Mollusca, Polyplacophora, new records, Costa Rica, Caribbean coast.

Houbrick (1968) realizó un inventario de los moluscos marinos del Caribe de Costa Rica, en la Provincia de Limón, identificó cinco especies de quitones en cuatro géneros y otros dos géneros de especies sin identificar: Acanthochitona rhodea (Pilsbry 1893), Acanthochitona sp., Ischnoplax pectinata (Sowerby 1840), Ischnochiton sp., Callistochiton sp., Chiton tuberculatus (Linnaeus 1758), Chiton marmoratus (Gmelin 1791) y Acanthopleura granulata (Gmelin 1791). Cincuenta años más tarde, la lista de quitones registrados para Costa Rica fue revisada (Schwabe \& Wehrtmann 2009). En esta lista actualizada se reconocen 24 especies de quitones para la costa Pacífica y ocho para el Caribe. Añaden a la lista de Houbrick (1968) a Ischnochiton erythronotus (C.B. Adams 1845), Stenoplax purpurascens (C.B. Adams 1845) y Stenoplax boogii
(Haddon 1886). Esta última especie se había informado también para la costa pacífica, en Isla de Cocos (Ferreira 1987a).

Slieker (2000), en su reseña de los quitones del mundo menciona 39 especies de quitones litorales y sublitorales en el Caribe. Para costas cercanas como las del Caribe colombiano se han documentado 22 especies (Gracia et al. 2005) y 21 para Venezuela (Bullock 1988, Bullock \& Franz 1994, Bullock et al. 1994). Schwabe \& Wehrtmann (2009) afirman que algunos micro-hábitats, como los fondos con acumulaciones de restos de coral, no han sido suficientemente explorados para la búsqueda de especies de quitones en Costa Rica. En este trabajo se presentan los resultados del examen del cascajo de coral sobre arena en la Provincia de Limón, con el objetivo de evidenciar la presencia de poblaciones que no 
habían sido registradas para la costa caribeña de Costa Rica.

\section{MATERIALES Y MÉTODOS}

Se realizaron recolectas de quitones en cuatro estaciones localizadas en la Provincia de Limón: tres cercanas al arrecife de franja en el Parque Nacional Cahuita (Playa Blanca, 9०44'14" N - 8250'19" W; Punta Cahuita, 9०44'51" N - 8248'39" W; Puerto Vargas, $9^{\circ} 44^{\prime} 40^{\prime \prime}$ N $-82^{\circ} 48^{\prime} 32^{\prime \prime}$ W) y otra en la Playa de Puerto Viejo $\left(9^{\circ} 39^{\prime} 33^{\prime \prime} \mathrm{N}-82^{\circ} 45^{\prime} 11.04\right.$ " W). La latitud y longitud de un punto cercano al centro de las estaciones se determinó usando un GPS. En las estaciones se recolectó a mano, examinando la superficie expuesta de las rocas, así como la superficie enterrada o en sombras. Se recolectó en profundidades menores a los dos metros. Cuando se trató de ejemplares con alta frecuencia sólo se recolectaron seis individuos. Los lotes de quitones recolectados fueron relajados y aplanados para su preservación usando alcohol etílico diluido con agua destilada al $70 \%$. Los organismos preservados fueron examinados, medidos y fotografiados utilizando un microscopio de disección Nikon SMZ1500. Algunos ejemplares fueron seleccionados para ser examinados con el microscopio electrónico de barrido (MEB). Estos se prepararon utilizando el método descrito en Bullock (1985). La determinación taxonómica de los quitones se realizó utilizando las guías taxonómicas de Kaas \& Van Belle (1985, 1987, 1990, 1994) y García-Ríos (2003). Se utilizó el esquema de clasificación de Kaas \& Van Belle (1998).

Los ejemplares fueron depositados en el Museo de Zoología de la Universidad de Costa Rica (MZUCR) y en la Colección Biológica de la Universidad de Puerto Rico en Humacao (CBUPRH).

\section{Nuevos Registros}

\section{Ischnochitonidae Dall 1889 \\ Callistochiton portobelensis Ferreira 1976}

Registros previos: Portobelo, Panamá y Key West, Florida (Kaas \& van Belle, 1994).

Registro nuevo: Dos ejemplares, Playa Blanca (944'14” N - 8250'19” W), Limón, Costa Rica; 11 de abril de 2009, zona entre mareas, bajo guijarros en pozas de $10 \mathrm{~cm}$ de profundidad; col M. Álvarez-Ruiz y C.I. García; CBUPRH 2693 (Fig.1) y CBUPRH 2692 (Fig. 2 y 3). Seis ejemplares, Punta Cahuita (9॰44'51" N - 8248'39” W), Parque Nacional Cahuita, Limón, Costa Rica; 6 de abril de 2009, 0.5 a $2 \mathrm{~m}$ profundidad; $100 \mathrm{~m}$ de la orilla; col M. Álvarez-Ruiz y C.I. García; CBUPRH 2755. Seis ejemplares, Puerto Vargas $\left(09^{\circ} 44^{\prime} 40^{\prime \prime} \mathrm{N}\right.$ - 8248'32" W), Parque Nacional Cahuita, Limón, Costa Rica; 9 de abril de 2009, 0.5 a $1.0 \mathrm{~m}$; bajo restos de coral parcialmente enterrado en arenas calcáreas, $30 \mathrm{~m}$ de la orilla; col M. Álvarez-Ruiz y C.I. García; MZUCR 7798; CBUPRH 2754.

Esta es una especie abundante bajo el cascajo de coral en zonas poco profundas. Se encontró en agregaciones de tres a cuatro individuos bajo rocas pulidas. A diferencia de las otras especies que aquí se presentan, $C$. portobelensis se suelta de la roca y se enrolla tan pronto es expuesta a la luz. Este comportamiento ha sido descrito para otras especies del género en el Pacífico (Eernisse et al. 2007). Kaas \& van Belle (1994) indican que esta especie presenta tegumento crema verdoso, con marcas irregulares más oscuras y solo ejemplares excepcionales son completamente rojos. De la pequeña muestra que se examinó $(\mathrm{N}=14)$ cuatro ejemplares presentaban coloración rojiza. Otros ejemplares avistados, pero no 


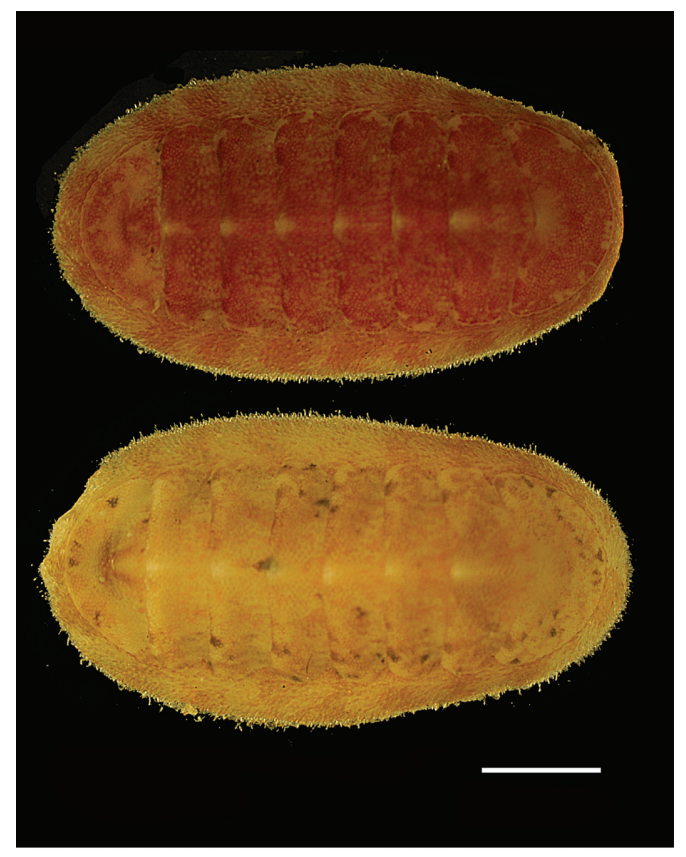

Fig. 1. Callistochiton portobelensis Ferreira, 1976, CBUPRH \#2693, longitud de la escala $=1 \mathrm{~mm}$.

Fig. 1. Callistochiton portobelensis Ferreira, 1976, CBUPRH \#2693, scale bar=1 mm.

recolectados sugieren que la coloración rojiza, al menos en estas estaciones en Costa Rica, no es excepcional (Fig. 1).

La rádula del ejemplar CBUPRH 2692 difiere de la descripción y los dibujos de cámara lúcida del holotipo (Ferreira 1976). El ejemplar observado tiene un diente central más estrecho en su extremo anterior, cerca de $15 \mu \mathrm{m}$ (Fig. 3A), a diferencia de los $30 \mu \mathrm{m}$ en la descripción del holotipo. Otra diferencia notable es la forma del diente lateral mayor, como se observa en la micrografía, en ese diente un extremo redondeado, con una protuberancia menor orientada hacia el centro de la rádula (Fig. 3B). El descrito por Ferreira (1976) tiene un extremo más filoso y un tubérculo de $25 \times 8 \mu \mathrm{m}$ que no se observa en nuestro ejemplar.

\section{Ischnochiton kaasi Ferreira 1987}

Registros previos: Bocas del Toro y cerca de Portobelo, Panamá (Ferreira 1987b).

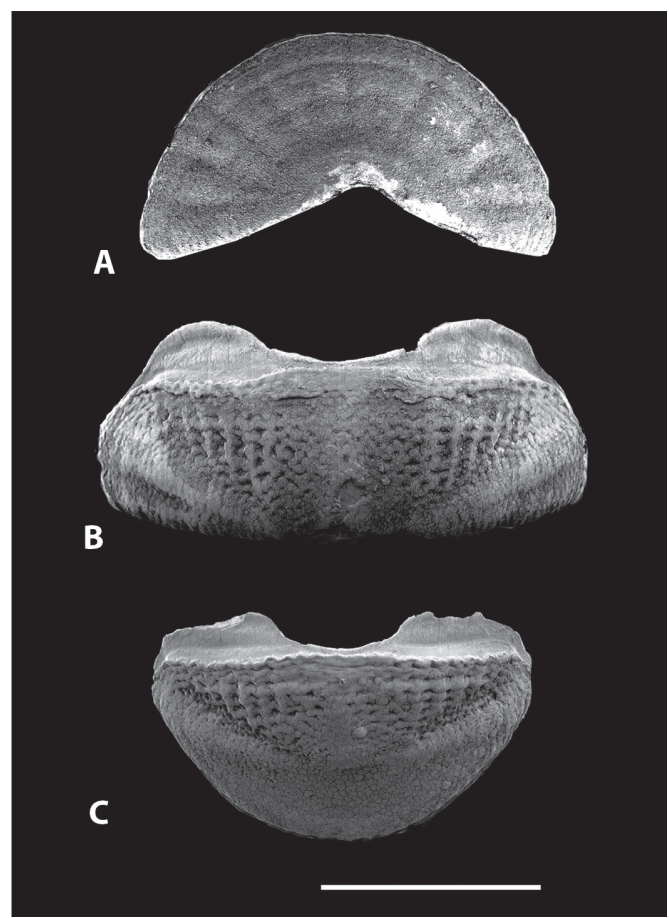

Fig. 2. Callistochiton portobelensis Ferreira, 1976, CBUPRH \#2692, longitud total del ejemplar 5.8mm; vista dorsal de: A, valva cefálica; B, valva intermedia IV; C, valva anal; longitud de la escala $=1 \mathrm{~mm}$.

Fig. 2. Callistochiton portobelensis Ferreira, 1976, CBUPRH \#2692, Whole specimen 5.8mm long, dorsal view of: A, head valve; $\mathrm{B}$, valve IV; $\mathrm{C}$, , tail valve; scale bar $=1 \mathrm{~mm}$

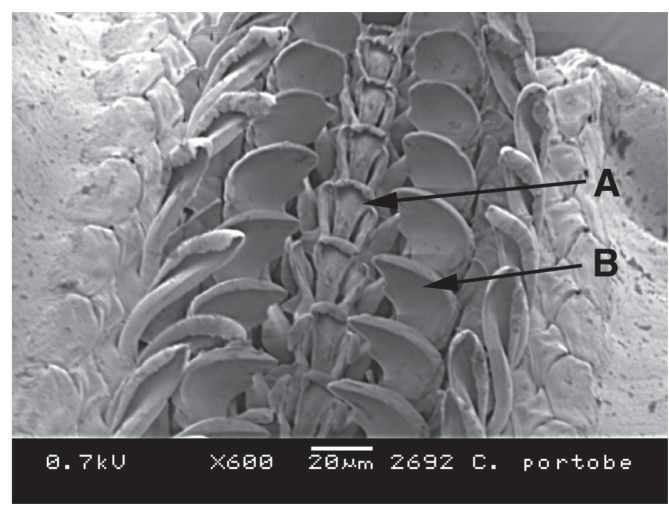

Fig. 3. Callistochiton portobelensis Ferreira, 1976, CBUPRH \#2692, rádula: $\mathrm{A}$, diente central; $\mathrm{B}$, diente lateral mayor.

Fig. 3. Callistochiton portobelensis Ferreira, 1976, CBUPRH \#2692, radulae: A, central tooth; B, major lateral tooth. 
Registro nuevo: Seis ejemplares, Punta Cahuita (9॰44'51" N - 8248'39" W), Parque Nacional Cahuita, Limón, Costa Rica; 6 de abril de 2009, $0.5 \mathrm{~m}$ profundidad; $100 \mathrm{~m}$ de la orilla; col M. Álvarez-Ruiz y C.I. García; CBUPRH 2746, CBUPRH 2691 (Fig. 4 y 5). Seis ejemplares, Puerto Vargas (09 44'40" N - 8248'32" W), Parque Nacional Cahuita, Limón, Costa Rica; 8 de abril de 2009, 0.5 a $1.0 \mathrm{~m}$; bajo restos de coral parcialmente enterrado en arenas calcáreas, $30 \mathrm{~m}$ de la orilla; col M. Álvarez-Ruiz y C.I. García; MZUCR 7799; CBUPRH 2756 y CBUPRH 2701 (Fig. 6). Tres ejemplares, Playa de Puerto Viejo (9³9’33” N - 8245'11.04” W), Limón, Costa Rica; 7 de abril de 2009, 1 a 2m profundidad, $80 \mathrm{~m}$ de la orilla; col M. Álvarez-Ruiz y C.I. García; CBUPRH 2745.

Esta es una especie muy común bajo el cascajo de coral posado sobre arena. Se puede distinguir de otras especies del género por la textura de su tegumento (Fig. 5), el ancho de las escamas dorsales del cinturón (90 a $115 \mu \mathrm{m})$ y la cantidad de ribetes que ornamentan esas escamas $(>17)$. Ischnochiton erythronotus, una especie muy similar, posee escamas dorsales de unos $150 \mu \mathrm{m}$ de ancho y alrededor de 12 costillas (García-Ríos 2003). Esta última especie, aunque registrada para Costa Rica, no fue encontrada en ninguna de las estaciones de colección. Los ejemplares de I. kaasi de Costa Rica muestran mayor variedad de color que la sugerida en Kaas \& Van Belle (1990). Eran comunes los ejemplares de tegumento color crema con marcas marrón alargadas longitudinalmente. Estos colores se extienden al cinto, que muestra bandas alternas de crema y marrón. Otros ejemplares presentan tegumento claro con marcas oscuras, algunos de ellos con el cinturón blanco excepto el área anterior, que está coloreada de negro sólido. Otros patrones con marcas gris verdosas están presentes.

La micrografía de la rádula del ejemplar CBUPRH 2701 permite apreciar un diente central estrecho, con ensanchamiento en la punta de cerca de $18 \mu \mathrm{m}$ (Fig. 6), casi la mitad de la medida de ese diente en el holotipo según

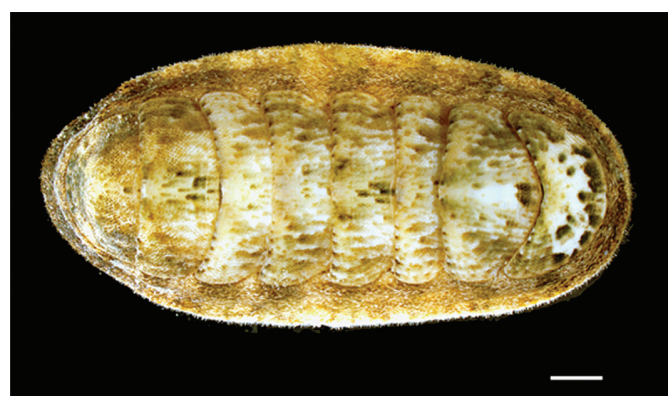

Fig. 4. Ischnochiton kaasi Ferreira, 1987, CBUPRH 2691, longitud de la escala $=1 \mathrm{~mm}$.

Fig. 4. Ischnochiton kaasi Ferreira, 1987, CBUPRH 2691, scale bar $=1 \mathrm{~mm}$.

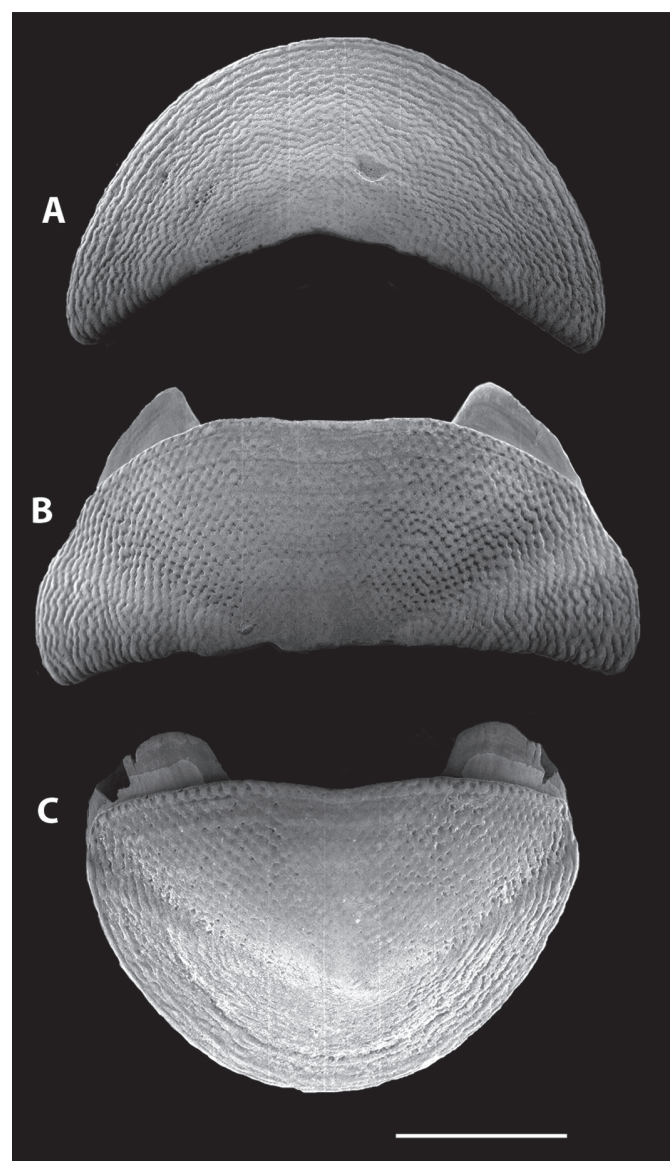

Fig. 5. Ischnochiton kaasi Ferreira, 1987, CBUPRH \#2691, longitud total del ejemplar $11.3 \mathrm{~mm}$, vista dorsal de: A, valva cefálica; $\mathrm{B}$, valva intermedia IV; $\mathrm{C}$, valva anal; longitud de la escala $=1 \mathrm{~mm}$.

Fig. 5. Ischnochiton kaasi Ferreira, 1987, CBUPRH \#2691, whole specimen $11.3 \mathrm{~mm}$ long, dorsal view of: A, head valve; $\mathrm{B}$, valve IV; $\mathrm{C}$, tail valve; scale bar $=1 \mathrm{~mm}$. 


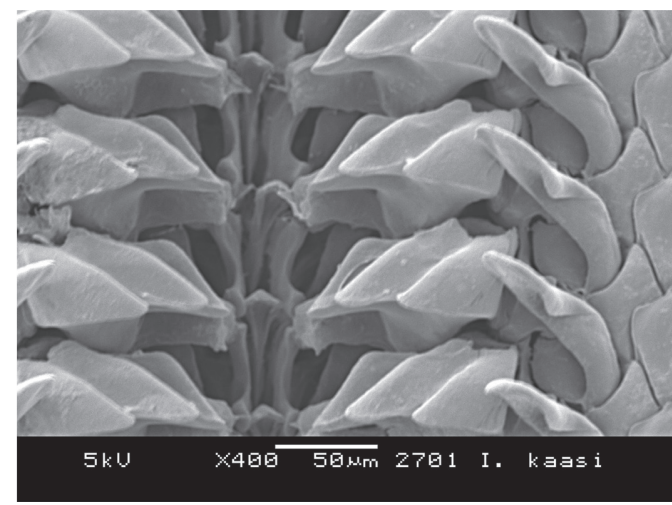

Fig. 6. Ischnochiton kaasi Ferreira, 1987, CBUPRH \#2701, rádula.

Fig. 6. Ischnochiton kaasi Ferreira, 1987, CBUPRH \#2701, radulae.

Ferreira (1987b). El diente lateral mayor es tricúspide.

\section{Ischnochiton pseudovirgatus Kaas 1972}

Registros previos: Jamaica, Curaçao, Aruba, Trinidad, Barbados, Florida (Kaas \& Van Belle 1990), Colombia (Gracia et al. 2005).

Registro nuevo: Dos ejemplares, Puerto Vargas (09 $44^{\prime} 40^{\prime \prime} \mathrm{N}-82^{\circ} 48^{\prime} 32^{\prime \prime} \mathrm{W}$ ), Parque Nacional Cahuita, Limón, Costa Rica; 8 de abril de 2009, 0.5-1.0m; bajo restos de coral parcialmente enterrado en arenas calcáreas, $10-15 \mathrm{~m}$ de la orilla; col M. Álvarez-Ruiz y C.I. García; MZUCR 7800; CBUPRH 2697 (Fig. 7).

Ischnochiton pseudovirgatus es una especie pequeña que alcanza hasta los $6 \mathrm{~mm}$ de longitud (Ferreira 1985). Es fácilmente reconocida por tener tegumento color blanco a crema con puntos azules sobre un fondo marrón a ambos lados de la región del yugo de muchas valvas (Fig. 7).

Acanthochitonidae Pilsbry 1893

Acanthochitona balesae Abbott 1954

Registros previos: Florida, Aruba, Trinidad (Kaas 1972), Bahama Grande, Panamá (Lyons 1988), Cuba y Puerto Rico (GarcíaRíos 2003).

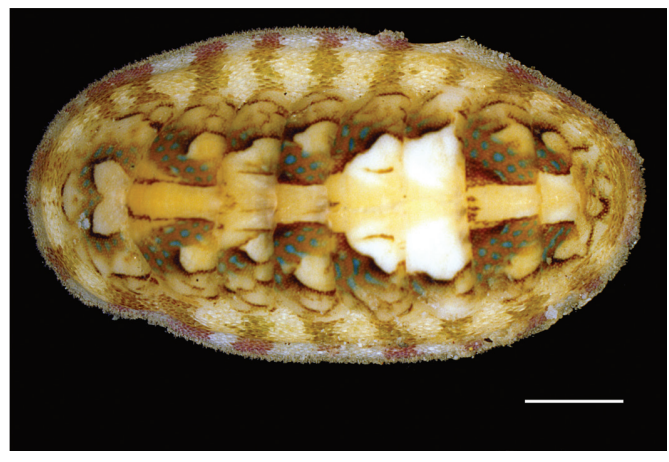

Fig. 7. Ischnochiton pseudovirgatus Kaas, 1972, CBUPRH 2697, longitud de la escala $=1 \mathrm{~mm}$.

Fig. 7. Ischnochiton pseudovirgatus Kaas, 1972, CBUPRH 2697, scale bar $=1 \mathrm{~mm}$.

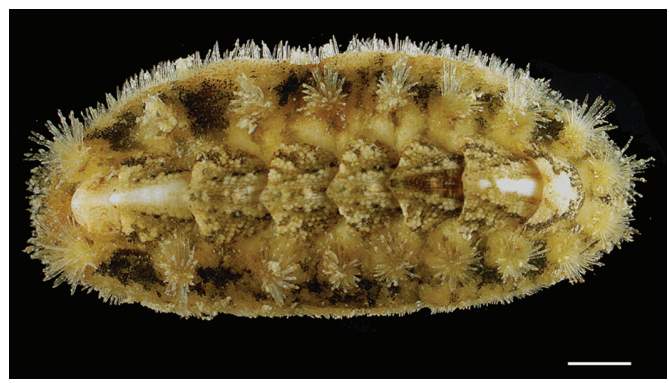

Fig. 8. Acanthochitona balesae Abbott, 1954, CBUPRH 2696, longitud de la escala $=1 \mathrm{~mm}$.

Fig. 8. Acanthochitona balesae Abbott, 1954, CBUPRH 2696, scale bar $=1 \mathrm{~mm}$.

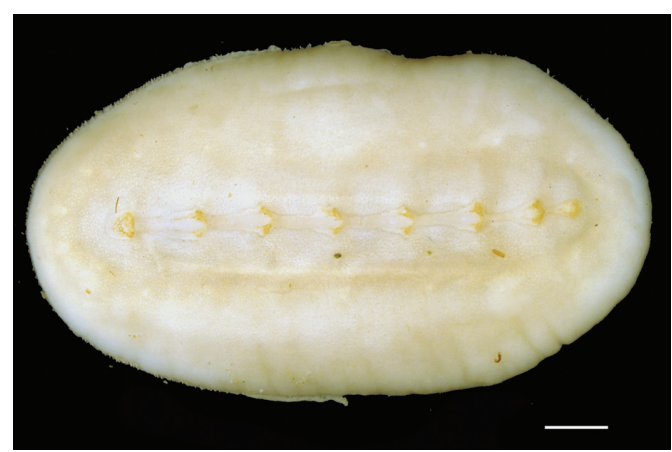

Fig.9. Cryptoconchus floridanus (Dall, 1889), CBUPRH 2695, longitud de la escala $=1 \mathrm{~mm}$.

Fig.9. Cryptoconchus floridanus (Dall, 1889), CBUPRH 2695, scale bar $=1 \mathrm{~mm}$. 
Registro nuevo: Un ejemplar, Puerto Vargas (0944'40" N - 82 48'32" W), Parque Nacional Cahuita, Limón, Costa Rica; 9 de abril de 2009, 0.5-1.0m; bajo restos de coral parcialmente enterrado en arenas calcáreas, 30m de la orilla; col M. Álvarez-Ruiz y C.I. García; CBUPRH 2696 (Fig. 8). Un ejemplar, Playa Blanca (9०44'14" N - 8250'19” W), Limón, Costa Rica; 11 de abril de 2009, zona entre mareas, bajo guijarros en pozas de $10 \mathrm{~cm}$ profundidad; col M. Álvarez-Ruiz y C.I. García; CBUPRH 2698.

Acanthochitona balesae es un quitón de apariencia alargada, que puede alcanzar los $10 \mathrm{~mm}$ de longitud. Son de coloración variada, la mayoría blancos con manchas crema, marrón o negras. Tienen 18 mechones de espículas en el cinturón, con unas 50 espículas por mechón. El área lateropleural de las valvas está cubierta con pústulos ovales de $90 \times 45 \mu \mathrm{m}$. El yugo ensancha en su área anterior y tiene márgenes irregulares (Lyons 1988). Los dos ejemplares que se registran para Costa Rica tienen 9.4 y $7.4 \mathrm{~mm}$ de longitud. Ambos tienen pústulos que alcanzan los $120 \mu \mathrm{m}$, algo mayores a los de la descripción citada. Algunas de las espículas de los mechones del cinturón están parcialmente teñidas de rojo.

\section{Cryptoconchus floridanus (Dall 1889)}

Registros previos: Cayos de la Florida: Key West, Cayo Largo, Dry Tortugas (los tres como localidad tipo), sureste de la Florida, Bahamas, Puerto Rico, Cuba, Jamaica, Islas Caimán, Aruba, Bonaire (Lyons 1988, Slieker 2000) y México (Reyes-Gómez \& SalcedoVargas 2002).

Registro nuevo: Un ejemplar, Puerto Vargas (0944'40" N - 8248'32" W), Parque Nacional Cahuita, Limón, Costa Rica; 8 de abril de 2009, 1.0m; bajo pedazo pulido de coral muerto, parcialmente enterrado en arenas calcáreas en zona de Thalassia testudinum, $15 \mathrm{~m}$ de la orilla; col M. Álvarez-Ruiz y C.I. García; CBUPRH 2695 (Fig. 9).
El único ejemplar encontrado de esta especie tenía el cinturón blanco. La coloración dominante de esta especie es marrón muy oscuro a negro. Kaas (1972) menciona haber examinados ejemplares con esa coloración en Bonaire. Las branquias están localizadas en la parte posterior del surco paleal (merobranquial) y se extienden hasta la mitad del cuerpo (bajo la valva IV), las últimas son más grandes (abanal).

Otras cuatro especies previamente reportadas para Costa Rica (Schwabe \& Wehrtmann 2009) fueron encontradas dentro de los límites del Parque Cahuita. Estas fueron: Ischnoplax pectinata (Sowerby 1840); Stenoplax purpurascens (C.B. Adams 1845); Acanthopleura granulata (Gmelin 1791) y Acanthochitona rhodea (Pilsbry 1893).

\section{DISCUSIÓN}

Con estos cinco registros aumentan a 13 las especies de quitones documentadas para el Caribe de Costa Rica. Los registros de $C$. portobelensis e I. kaasi eran esperados, pues son especies que se conocían para la costa caribeña de Panamá (Schwabe \& Wehrtmann 2009). Es muy probable que Houbrick (1968), al registrar los ejemplares de Callistochiton sp. e Ischnochiton sp. se refiriera a las especies $C$. portobelensis e I. kaasi que fueron descritas ocho años después por Ferreira (1987b). Aunque Houbrick (1968) indica que estas especies eran raras en las estaciones que él estudió, son muy comunes en el litoral de Cahuita.

Esta colección documenta nueve quitones en las cercanías del arrecife en el Parque Nacional Cahuita, casi el $70 \%$ de la totalidad registrada para el Caribe de Costa Rica. Con estos registros se supera en uno la cantidad de especies descritas para Panamá (Lyons 1988, Kaas 1972, Ferreira 1976, 1987b, Bullock 1985, 1988). Trabajos adicionales, que incluyan la fauna asociada a los fondos líticos más profundos, así como costas rocosas de mayor energía a las examinadas aquí, deberían evidenciar una diversidad de quitones aún mayor. 


\section{AGRADECIMIENTOS}

Una licencia sabática otorgada por la Universidad de Puerto Rico en Humacao permitió, al primer autor, completar este manuscrito. El Fondo de Investigación Institucional de la Universidad de Puerto Rico en Ponce permitió realizar el trabajo de campo a la segunda autora. El equipo de microscopía fue facilitado por el Programa Partnership for Research \& Education in Materials de la UPR-Humacao (NSFDMR-0934195). Agradecemos la orientación y recomendaciones sobre lugares para obtener muestras a Ingo S. Wehrtmann de la Universidad de Costa Rica y las críticas al manuscrito de María Ramos Santiago, Ariel Díaz y tres revisores anónimos.

\section{RESUMEN}

Los poliplacóforos asociados a los arrecifes de coral en la costa caribeña de Costa Rica han sido poco estudiados. El examen del cascajo de coral acumulado en el sublitoral somero, en cuatro estaciones de colección, localizadas en la Provincia de Limón reveló una diversidad de quitones mayor a la documentada. Anteriormente se habían registrado ocho especies para el Caribe costaricense: Ischnochiton erythronotus (C.B. Adams, 1845); Ischnoplax pectinata (Sowerby 1840); Stenoplax boogii (Haddon, 1886); S. purpurascens (C.B. Adams, 1845); Acanthopleura granulata (Gmelin, 1791); Chiton marmoratus Gmelin, 1791; C. tuberculatus Linnaeus, 1758; Acanthochitona rhodea (Pilsbry, 1893). Otras cinco se registran aquí por primera vez: Callistochiton portobelensis Ferreira 1976; Ischnochiton kaasi Ferreira 1987; I. pseudovirgatus Kaas 1972; Acanthochitona balesae Abbott 1954; Cryptoconchus floridanus (Dall 1889).

Palabras clave: Mollusca, Polyplacophora, nuevos registros, Costa Rica, costa Caribe.

\section{REFERENCIAS}

Bullock, R.C. 1985. The Stenoplax limaciformis (Sowerby, 1832) species complex in the New World (Mollusca: Polyplacophora: Ischnochitonidae). Veliger 27: 291-307.

Bullock, R.C. 1988. The genus Chiton in the new world (Polyplacophora: Chitonidae). Veliger 31: 141-191.

Bullock, R.C. \& C.J. Franz. 1994. A preliminary taxonomic survey of the chitons (Mollusca: Polyplacophora) of
Isla Margarita. Nueva Esparta, Venezuela. Sociedad de Ciencias Naturales La Salle, tomo LIV, $\mathrm{N}^{\circ} 141$, 222: 9-49.

Bullock, R.C., C.J. Franz \& J. Buitrago. 1994. A report on a collection of chitons (Mollusca: Polyplacophora) dredged near Isla Coche, Nueva Esparta, Venezuela. Sociedad de Ciencias Naturales La Salle, tomo LIV, $\mathrm{N}^{\circ}$ 141, 220: 77-93.

Eernisse, D.J., R.N. Clark \& A. Draeger. 2007. Polyplacophora, p. 701-713. In J.T. Carlton (ed.). Light and Smith Manual: Intertidal Invertebrates from Central California to Oregon. University of California, Berkeley, California, EEUU.

Ferreira, A.J. 1976. A new species of Callistochiton in the Caribbean. Nautilus 90: 46-49.

Ferreira A.J. 1985. Chiton (Mollusca: Polyplacophora) fauna of Barbados, West Indies, with the description of a new species. Bull. Mar. Sci. 36: 189-219.

Ferreira, A.J. 1987a. The chiton fauna of Cocos Island, Costa Rica (Mollusca: Polyplacophora) with the description of two new species. Bull. South Cal. Acad. Sci. 86: 41-53.

Ferreira, A.J. 1987b. Two new species of Ischnochiton (Mollusca: Polyplacophora) in the western central Atlantic. Bull. Mar. Sci. 40: 145-151.

García-Ríos, C.I. 2003. Los quitones de Puerto Rico. Isla Negra Editores, San Juan, Puerto Rico.

Gracia A., J.M. Díaz \& N.E. Ardila. 2005. Quitones (Mollusca: Polyplacophora) del Mar Caribe Colombiano. Biota Colombiana 6: 117-125.

Houbrick, J.R. 1968. A survey of the littoral marine mollusks of the Caribbean coast of Costa Rica. Veliger 11: 4-23.

Kaas, P. 1972. Polyplacophora of the Caribbean region. Stud. Fauna Curaçao 41: 1-162.

Kaas, P. \& R.A. Van Belle. 1985. Monograph of living chitons (Mollusca: Polyplacophora). Suborder Ischnochitonina: Ischnochitonidae: Schizoplacinae, Callochitoninae and Lepidochitoninae. E.J. Brill, Leiden, Holanda.

Kaas, P. \& R.A. Van Belle. 1987. Monograph of living chitons (Mollusca: Polyplacophora. Suborder Ischnochitonina: Ischnochitonidae: Chaetopleuridae, Ischnochitoninae. E.J. Brill, Leiden, Holanda. 
Kaas, P. \& R.A. Van Belle. 1990. Monograph of living chitons (Mollusca: Polyplacophora). Suborder Ischnochitonina: Ischnochitonidae: Ischnochitoninae. E.J. Brill, Leiden, Holanda.

Kaas, P. \& R.A. Van Belle. 1994. Monograph of living chitons (Mollusca: Polyplacophora), Vol. 5, Suborder Ischnochitonina: Ischnochitonidae. E.J. Brill, Leiden, Holanda.

Kaas, P. \& R.A. Van Belle. 1998. Catalogue of living chitons (Mollusca: Polyplacophora). Backhuys, Leiden, Holanda.

Lyons, W.G. 1988. A review of Caribbean Acanthochitonidae (Mollusca: Polyplacophora) with descriptions of six new species of Acanthochitona Gray, 1821. Am. Malac. Bull. 6:79-114.

Reyes-Gómez, A. \& M.A. Salcedo-Vargas. 2002. The recent mexican chiton (Mollusca: Polyplacophora) species. Festivus 34: 17-27.

Schwabe, E. \& I.S. Wehrtmann. 2009. Chitons, p. 323-331. In I.S Wehrtmann \& J. Cortés (eds). Marine Biodiversity of Costa Rica, Central America. Monographiae Biologicae 86. Springer and Business Media B.V., Berlin, Alemania.

Slieker, F.J.A. 2000. Chitons of the world, an illustrated synopsis of recent Polyplacophora. L'Informatore Piceno, Italia. 